\title{
Stationary Phase Physiology Tell-Tales the Regulation of Bioactive Metabolite Production in Bacteria
}

\author{
Matsobane G Tlou ${ }^{1 *}$ and Mampolelo M Photolo ${ }^{2}$ \\ ${ }^{1}$ Department of Biochemistry, North-West University, South Africa \\ ${ }^{2}$ Department of Biochemistry, University of Johannesburg, South Africa
}

*Corresponding author: Matsobane G Tlou, Department of Biochemistry, School of Physical and Chemical Sciences, North-West University, Mafikeng Campus, South Africa.

To Cite This Article: Matsobane G Tlou. Stationary Phase Physiology Tell-Tales the Regulation of Bioactive Metabolite Production in Bacteria. 2020 - 9(4). AJBSR.MS.ID.001411. DOI: 10.34297/AJBSR.2020.09.001411.

Received: 耕 July 06, 2020; Published: 紫 July 10, 2020

\section{Opinion}

Bacteria exist in a variety of physiological types, environmental habitats and niches of occurrence. In these environments, bacteria are predominantly found in the stationary growth-phase (SGP) due to oligotrophic conditions, biotic and physico-chemical stress [1]. The physiological and biochemical properties of bacteria reflect their "environmental-fitness" or adaptability to the environmental habitats i.e. extreme barophilic bacteria isolated from the deep-sea [2], a thermophilic isolate from an ultra-deep South African gold mine [3], metalophillic species from a domestic showerhead biofilm [4] plant/animal symbiotic bacteria [5,6]. The ability to survive and proliferate under such conditions depends on the physiological regulation of SGP and consequently, the synthesis of natural products/bacterial bioactive metabolites (BBM) [7], a process intricate to the global stress-response mechanism in bacteria [8]. This therefore indicates that BBM synthesis can influence ecological landscapes through direct antagonism, niche defence and signalling [9]. Albeit the ecological significance, bacterial natural products have been used for example as anticancer drug leads and other human diseases and are the main source of clinically used antimicrobials to combat infectious diseases [9]. It is therefore, the opinion of the authors that, bacterial metabolites (BM) production can be exploited for the purposes of directed drug discovery i.e.

(i) The production by marine bacteria of specialized lipids (for lipid metabolism-associated diseases) in response to changes in salinity, temperature and pressure [7],

(ii) Siderophore production (for diseases in metal-ion imbalance and drug delivery systems) in response to limiting concentrations of micronutrients (Fe3+ in particular) [7], (iii) Chemical communication (antibiotics and cell signaling ) upon co-cultivation of two or more bacterial species [10] and,

(iv) The production of "plant-derived" antimicrobials and radical scavenging metabolites (for antimicrobial and anticancer therapy) by bacterial symbionts $[5,10]$.

The bacterial bioactive metabolites are low molecular weight (100-1000 Da) natural products of overwhelming chemical diversity [9] and consequently, diverse biological activity. The enzymes that catalyses the reactions of molecular assembly (metabolite synthesis) are encoded on biosynthetic gene clusters (BGCs) and Next Generation Sequencing (NGS) continues to reveal an unprecedented potential for BBM production in bacteria [11]. This potential remains untapped because, In vitro activation for most of the (cryptic or silent) BGC's has proven to be challenging in many laboratories (and for a diversity of reasons) albeit some success stories reported in literature [7].

At this point, the author would also like to extend on the opinion presented earlier and suggest that the manipulation SGP physiology in a stress-antagonistic manner can result in the activation of specific BCGs and can be exploited in order to inform targeted bioactivity or directed prospecting for therapeutics. We therefore present herein, a few aspects of SGP physiology that tell-tales of the key considerations in the regulation of BMM synthesis.

\section{Stationary Growth-Phase Physiology, BGC Activation and Metabolite Synthesis}

It can be easily inferred from the literature that stress-antagonism is one of the key triggers to bacterial bioactive metabolite pro- 
duction [7-10]. Furthermore, since BBM production is tightly linked to the stationary phase/global stress response, the role played by alternative sigma factors and stationary phase cis-acting regulatory sequences in this regard is also documented [12]. Therefore, we hypothesize that the above-mentioned factors can drive basal BGC expression and that high-level and (or) differential activation is achievable with exposure to a particular stimulus or a combination thereof.

A case in point is the reported metabolite-mediated bacterial responses to conditions of high salinity, low temperature, oligotrophy and pressure reported for marine bacteria [10]. The ability of bacteria to maintain biological functions under the stressful conditions results from changes in protein, sterol, hopanoid and carotenoid content, but mainly from changes in membrane lipid composition [13] or the production of specialized lipids such as sterols and polyunsaturated fatty acids $[14,15]$. Bacterial cells are also able to produce fatty acids as niche defense compounds. For example, Pseudoalteromonas haloplanktis produces isovaleric acid (3-methylbutanoic acid) and 2-methylbutyric acid (2-methylbutanoic acid), which have antibacterial activities [16]. This, therefore, potentially ties the activation of the BCGs involved to bacterial fatty acid metabolism and the activities of the associated regulatory cisand trans-acting elements [17].

In some bacteria, carbon catabolite repression (CCR), a global regulatory mechanism for sequential utilization of carbon sources [18], has been linked to the regulation of bioactive metabolite synthesis and also reported as a key factor in the disease development for many pathogenic bacteria [18]. This therefore suggests CCR as a key regulatory mechanism in bacteria niche protection and also further points to the involvement of 'stress-inducible transcriptional programming' which tells-a-tale of the potential to differentially activate BCGs and perhaps, the involvement of epigenetic gene regulation [19] in metabolite-mediated stress response.

In conclusion, in a recent publication [20] reported on the adaptation of bacteria to oxidative stress by non-lethal exposure to H2O2 which amongst other possibilities could suggest the activation of radical scavenging BGCs and indicates the prospects for directed prospecting for antitumor therapy [21] and numerous other therapeutic metabolites in this or a related way. This coupled to the fact that bacteria are generally amenable to manipulation in the laboratory means that the SGP can be induced with ease and the physiology manipulated towards the production of the desired bioactivity.

\section{Acknowledgment}

None.

\section{Conflict of Interest}

No conflict of interest.

\section{Reference}

1. Nystrom T (2004) Stationary-phase physiology. Annu Rev Microbiol 58: 161-181.

2. Kato C, L Li, Nogi Y, Nakamura Y, Tamaoka J, et al. (1998) Extremely barophilic bacteria isolated from the Mariana Trench, Challenger Deep, at a depth of 11,000 meters. Appl Environ Microbiol 64(4): 1510-1513.

3. DeFlaun MF, Fredrickson JK, Dong H, Pfiffner SM, Onstott TC, et al (2007) Isolation and characterization of a Geobacillus thermoleovorans strain from an ultra-deep South African gold mine. Syst Appl Microbiol 30(2): 152-164.

4. Charnock C, Nordlie A L (2016) Proteobacteria, extremophiles and unassigned species dominate in a tape-like showerhead biofilm. Braz J Microbiol 47(2): 345-351.

5. Photolo M, Vuyo Mavumengwana, Lungile Sitole, Matsobane G Tlou (2020) Antimicrobial and Antioxidant Properties of a Bacterial Endophyte, Methylobacterium radiotolerans MAMP 4754, Isolated from Combretum erythrophyllum Seeds. Int J Microbiol 2020: 9483670.

6. Sharon G, Neha Garg, Justine Debelius, Rob Knight, Pieter C Dorrestein, et al. (2014) Specialized metabolites from the microbiome in health and disease. Cell metab 20(5): 719-730.

7. de Carvalho CC, Fernandes P (2010) Production of metabolites as bacterial responses to the marine environment. Mar Drugs 8(3): 705-727.

8. Abranches, Alaina R Martinez, Jessica K Kajfasz, Violeta Chávez, Danielle A Garsin, et al. (2009) The Molecular Alarmone (p)ppGpp Mediates Stress Responses, Vancomycin Tolerance, and Virulence in Enterococcus faecalis. J Bacteriol 191 (7): 2248-2256.

9. Chevrette MG, Karina Gutiérrez García, Nelly Selem Mojica, César Aguilar Martínez, Alan Yañez Olvera, et al. (2020) Evolutionary dynamics of natural product biosynthesis in bacteria. Nat Prod Rep 37(4): 566-599.

10. Crawford JM, Clardy J (2011) Bacterial symbionts and natural products. Chem Commun (Camb) 47(27): 7559-7566.

11. Petersen LE, Kellermann MY, Schupp PJ, Jungblut S, Liebich V, Bode Dalby M (2020) Secondary Metabolites of Marine Microbes: From Natural Products Chemistry to Chemical Ecology. YOUMARES 9-The Oceans: Our Research, Our Future. Springer, Cham.

12. Jaishankar J, Preeti Srivastava (2017) Molecular basis of stationary phase survival and applications. Front Microbiol 8: 2000.

13. Weber FJ, de Bont JA (1996) Adaptation mechanisms of microorganisms to the toxic effects of organic solvents on membranes. Biochim Biophys Acta 1286(3): 225-245.

14. Fabregas J, J Arán, Morales ED, Lamela T, Otero A (1997) Modification of sterol concentration in marine microalgae. Phytochemistry 46: 11891191.

15. Yano Y, A Nakayama, K Yoshida (1997) Distribution of polyunsaturated Fatty acids in bacteria present in intestines of deep-sea fish and shallow-sea poikilothermic animals. Appl Environ Microbiol 63(7): 25722577.

16. Hayashida Soiza G (2008) Purification and characterization of antibacterial substances produced by a marine bacterium Pseudoalteromonas haloplanktis strain. J Appl Microbiol 105(5): 1672-1677.

17. Fujita Y (2007) The regulation of fatty acid metabolism. Mol Microbiol 66(4): 829-839

18. Görke B, Stülke J (2008) Carbon catabolite repression in bacteria: many ways to make the most out of nutrients. Nat Rev Microbiol 6: 613-624.

19. Casadesús J, Low D (2006) Epigenetic Gene Regulation in the Bacterial World. Microbiol Mol Biol Rev 70 (3): 830-856.

20. Rodriguez Rojas A, Joshua Jay Kim, Paul R Johnston, Olga Makarova, Murat Eravci, et al. (2020) Nonlethal exposure to H2O2 boosts bacteri- 
al survival and evolvability against oxidative stress. PLoS Genet 16(3): e1008649.

21. Samudrala PK, Bibin Baby Augustine, Eshvendar Reddy Kasala, Lakshmi
Narendra Bodduluru, Chandana Barua, et al. (2015) Evaluation of antitumor activity and antioxidant status of Alternanthera brasiliana against Ehrlich ascitescarcinoma in Swiss albino mice. Pharmacognosy Res 7(1): 66-73. 